\title{
Dark mode metasurfaces: sensing optical phase difference with subradiant modes and Fano resonances
}

\author{
Ann RoberTs ${ }^{1 *}$, TIMOthy J Davis ${ }^{1}$ And DANIEl E GoMeZ ${ }^{2}$ \\ ${ }^{1}$ School of Physics, The University of Melbourne, 3010, Australia \\ ${ }^{2}$ Centre of Excellence for Exciton Science, RMIT University, GPO Box 2476, Melbourne VIC 3001 Australia \\ *Corresponding author: ann.roberts@unimelb.edu.au
}

Received XX Month XXXX; revised XX Month, XXXX; accepted XX Month XXXX; posted XX Month XXXX (Doc. ID XXXXX); published XX Month XXXX

We computationally investigate the excitation of subradiant modes and Fano resonances of arrays of simple antenna elements with subwavelength dimensions. We show that periodic arrangements of dipoles on a flat surface provide a highly flexible approach for developing a range of metasurfaces with high sensitivity to both the wavelength of the incident radiation and angle of incidence. This provides a platform for the development of a range of metasurfaces with a wavelength-tunable sensitivity to phase gradients in an incident field.

OCIS codes: (050.0050) Diffraction and gratings; (050.6624)

Subwavelength structures; (310.6628) Subwavelength structures, nanostructures.

http://dx.doi.org/10.1364/JOSAB.99.099999

Over the past decade, scattering of visible and near-infrared light by metallic nanoparticles has been one of the cornerstones of the field of plasmonics [1]. More recently, 'metasurfaces' consisting of periodic arrangements of nanoparticles acting as optical antennas that take advantage of the resonant properties of the constituent nanoparticles [2,3] have been proposed and demonstrated. These arrays of antennas with subwavelength dimensions can be used as the basis for a wide variety of photonic devices [4]. Arrays of simple dipoles can be used as the basis for filters and polarizers, pairs of antennas (dimers) have been used as sensors and various arrangements of three wires (trimers) have been shown to act as plasmonic 'rulers' or to perform information processing operations [5-12]. Frequencyselective surfaces also have a long history as spectral filters and other optical elements for use with far-infrared and millimetre waves [13-15] and many metasurface designs can be considered as optical analogs of these devices. Both metasurfaces and frequency-selective surfaces exhibit complex resonant behavior as a result of coupling between the particles and interference between different modes of the unit cell and diffracting plane waves. Here we investigate metasurfaces for sensing optical phase differences. Previous research in this area has shown that arrays of dolmen structures [16] are sensitive to the component of the phase gradient in an incident plane wave along a specific direction. This was extended to a metasurface where the unit cell consisted of an expanded arrangement of nanoparticles [17] that sensed phase difference along both directions in the plane of the surface. A wavevector-selective metasurface for $\mathrm{THz}$ radiation has also been demonstrated [18].

The fundamental resonance mode of a localized surface plasmon on a metal nanoparticle is usually dominated by the dipole moment of the oscillating charge providing a motivation for representing LSP resonances as simple dipole antennas. Here we consider scattering from two-dimensional, periodic arrangements of perfectly electrically conducting (PEC) wires where the unit cell is composed of three dipole antennas, a trimer, that can have various sizes, locations and orientations. The problem of scattering by multiple parallel conducting plates in a unit cell has been considered previously [19] with a view to producing frequency-selective surfaces with multiband operation. More recently the influence of the substrate on the optical properties of metasurfaces with unit cells composed of multiple antenna elements that exhibit Fano resonances was investigated [20]. Here we computationally demonstrate that periodic ensembles of subwavelength dipoles can be arranged to be sensitive to phase variations in the incident field. The unit cells consist of nanoparticle trimers which are shown to exhibit plasmonic dark modes and Fano resonances. We look at unit cells consisting of three parallel antennas (Fig. 1(b)) and three dipoles arranged in a triangle (Fig. 1(c)). Both these structures exhibit dark modes, including magnetic modes, that can be excited using an off-normally incident plane wave. We also revisit metasurfaces composed of dolmen arrangements of antennas (Fig. 1(d)) and demonstrate scattering into a crosspolarized field component that is sensitive to angle of incidence, i.e. to phase differences in the incident field [16].

Although a wide variety of methods, including full-field techniques, exist for studying scattering by nanostructures, the computational approach used here follows a conventional modal method [13, 14, 20, 21], also known as the modalmatching technique, that has been used with considerable success to describe a range of frequency selective surfaces [21]. A complete description of the modal method can be found in [21-23], its extension to the role played by substrates in [24] 
and its use with multi-element unit cells in [20], so the technique is only summarized here for completeness. The electric and magnetic fields above and below a periodic array of subwavelength structures are written as a discrete sum over transverse electric (TE) and transverse magnetic (TM) Floquet modes. If we assume that the structures are infinitesimally thin and PEC, the surface currents excited on these structures can be written as a sum over orthonormal functions of two variables satisfying the appropriate boundary conditions at the edges of the plates. By looking at the coupling between each of these modes and the diffracted plane wave Floquet components, a series of linear equations for the modal amplitudes can be derived and solved using standard computational methods. Once these modal amplitudes are known, the amplitudes of all propagating and evanescent plane waves can be determined, along with the total reflected and transmitted power.

Specifically, we consider here an infinite, square twodimensional array of period, $d$, occupying the $x-y$ plane where each unit cell consists of ensembles of infinitesimally thin, PEC dipoles lying in the plane. The assumption of perfect conductivity is widely used when considering frequencyselective surfaces for use in the far-infrared and millimeter wave regions of the electromagnetic spectrum. The wires are all identical, having length $a$ and width $b$ and we assume that the substrate has a refractive index of 1 . The methodology, however, is easily extended to include antennas of different lengths, the presence of a finite thickness [25] or semi-infinite substrates $[24,26]$ or for arrays not exhibiting square symmetry. The surface is illuminated by a monochromatic plane wave of wavenumber $k_{0}=2 \pi / \lambda$ where $\lambda$ is the wavelength incident from vacuum at an angle to the surface normal of $\theta$. (a)

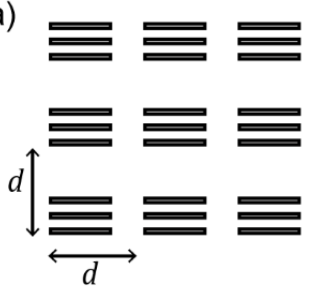

(c)

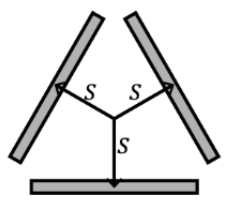

(b)

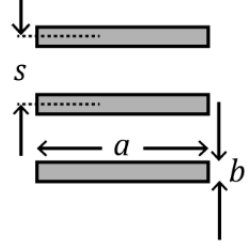

(d)

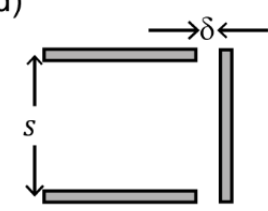

Fig. 1. The surface under consideration is an infinite square array of period $d$ with unit cells composed of several dipoles of length $a$ and width $b$. Specific unit cells considered here comprise (b) three identical parallel wires with distances between their centers given by $s$, (c) three wires arranged in a symmetric triangular configuration with the distance from the center of the wire to the geometric center of the ensemble given by $s$ and (d) a dolmen structure as shown.

In many cases, the currents on the antennas are welldescribed by the lowest order current mode so that the monomodal approximation [24, 27], referred to as the 'minimal model' in [20], can be used, thereby facilitating theoretical interpretation, significantly enhancing numerical performance and, thus, extending the range of problems and parameter space that can be investigated. Although each antenna is assumed to carry a current with the same spatial distribution, they each have a different amplitude due to variations in coupling of each wire to the incident field and to differences in intra-cell coupling between wires. We, therefore, write the standing-wave surface current on the wires in terms of a simple sinusoidal expression enforcing the condition that the current goes to zero at the ends of the wire and is uniform across the direction perpendicular to the direction of the current. Assuming the wire is oriented parallel to a unit vector $\hat{\alpha}$ and is centered on the point in the $x-y$ plane $\vec{x}_{T m}$, the current on antenna $m$ will have a spatial dependence on transverse coordinates, $\vec{x}_{T}=(x, y)$ given by:

$$
\vec{\Psi}_{m}\left(\vec{x}_{T}\right)=\left\{\begin{array}{cc}
\sqrt{\frac{2}{a b}} \cos \left[\pi\left(\vec{x}_{T}-\vec{x}_{T m}\right) \cdot \hat{\alpha} / a\right] \hat{\alpha} & \text { on dipole } \\
0 & \text { elsewhere }
\end{array} .\right.
$$

Assuming the dipoles do not overlap, the total surface current $\vec{K}\left(\vec{x}_{T}\right)$ across one unit cell can then be written as a sum over each antenna:

$$
\vec{K}\left(\vec{x}_{T}\right)=\sum_{m} B_{m} \vec{\Psi}_{m}\left(\vec{x}_{T}\right)
$$

where $B_{m}$ is the amplitude of the current distribution on wire $m$.

By applying the boundary conditions requiring: (i) continuity of the transverse component of the electric field across the boundary defined by the surface, (ii) vanishing amplitude of this field on the PEC antennas, and (iii) that the discontinuity in the transverse component of the magnetic field is related to the surface current, gives rise to a set of linear equations that can be solved for the amplitudes $B_{m}$ :

$$
\sum_{m} Q^{m M} B_{m}=D^{M}
$$

where

$$
Q^{m M}=\frac{1}{2} \sum_{r p q} Z_{r p q} C_{r p q}^{m} C_{p q r}^{M^{*}}
$$

and

$$
D^{M}=-2 \sum_{r} E_{r 00}^{0} C_{00 r}^{M^{*}} .
$$

In Eqs (4) and (5) the index $r$ denotes either TE ( $r=1)$ or TM ( $r=$ 2) polarization and the indices $p$ and $q$ characterize the transverse spatial frequencies of each diffracted Floquet mode. The quantity $E_{r 00}^{0}$ is the amplitude of the incident Floquet mode with polarization $r$, and $Z_{r p q}$ is the mode impedance of mode $r p q$ [21]. In Eqs. (4) and (5) the asterisk denotes complex conjugation and the terms $C_{r p q}^{m}$ in Eq. (4) describe the coupling of the current on antenna $m$ to the plane wave mode $r p q$ and depend on the specific arrangement of wires under consideration. The off-diagonal terms in $Q^{m M}$ characterize coupling between particles $m$ and $M$, whereas the diagonal terms describe direct coupling of each particle to the fields. Note that the array, $Q^{m M}$ depends only on the geometry of the problem and the dielectric environment whereas $D^{M}$ also depends on the incident field. Once these quantities are known, it is possible to find the amplitudes $B_{m}$ using eqn (3) and then the amplitudes of each Floquet (plane-wave) mode, $E_{r p q}$, using an expression obtained from the boundary conditions: 


$$
E_{r p q}=-\frac{Z_{r p q}}{2} \sum_{m} B_{m} C_{r p q}^{m}
$$

This then permits the calculation of the fields above and below the metasurface and, hence, the reflected and transmitted power. In our numerical implementation, the sum appearing in Eq. (4) is truncated and all results presented below include 43 values of both $p$ and $q$. Since here we consider unit cells with three antennas, solving Eq. (3) involves simply solving a set of three linear equations for the three amplitudes, i.e. inverting a $3 \times 3$ matrix.

The method is easily applicable to a wide variety of ensemble configurations, but here we will focus on three different configurations, each consisting of a unit cell containing three identical antennas to illustrate the flexibility afforded by using antenna ensembles. These are illustrated in Fig. 1. The specific cases are Fig. 1(b) where the unit cell consists of three parallel wires, another Fig. 1(c) where three wires form a triangle and a third Fig. 1(d) consisting of a dolmen structure. Although, as outlined above, this approach uses only one current mode on each wire, reference [20] demonstrates good agreement with the results of this model using a complete set of current modes and with calculations obtained using the Finite Element Method (FEM). It should be noted that this method is considerably faster, by several orders of magnitude, than the FEM. In our implementation of this method in MATLAB (2016a) on a standard PC (Intel i7-4600U CPU, 2.10GHz, 8GB RAM), the calculation time for a single point in the plots shown in Figs. 2-4 is of the order of few milliseconds. Running a comparable calculation using COMSOL Multiphysics V5.2a on the same computer leads to a computation time of approximately 1 minute, depending on the specifics of the model. Even considering that the FEM faces particular challenges in modelling infinitesimally thin PEC plates, there are very significant computational savings in using the modal method. Furthermore, as seen in Eq. (3), the determination of the current amplitude and phase is integral to this approach providing considerable insight into the underlying physics as shown in the calculations presented below.

We first consider a unit cell composed of three parallel dipoles separated by a distance $s$. Since the antennas are assumed perfectly conducting, the array period, $d$, provides a natural scaling parameter for all lengths. Fig. 2 shows the normalized reflectance from an array of wires with dimensions $a=0.70 \mathrm{~d}$ and $b=0.02 d$ and with separation $s=0.05 d$. The small value of $b$ minimizes the discrepancy between the monomodal method and rigorous techniques and is also consistent with considering all antennas to be simple dipoles. The incident field is assumed to be a plane wave with electric field polarized parallel to the antennas and results for both normal incidence (Fig. 2(a) and (b)) and TE-polarized illumination incident at $30^{\circ}$ to the normal (Fig. 2(c) and (d)) are shown. It is also instructive to compare the reflectance from an array of single antennas of the same dimensions and the ensemble consisting of three parallel wires as shown in Fig. 2(a) and (c).

The reflectance spectra shown in Fig. 2 are complex. At normal incidence (Fig. 2(a)) a single antenna produces a broad peak in reflectance centered on a wavelength of $1.45 d$, but in the case of the trimer, this feature is modified by the appearance of a Fano resonance located near $1.43 d$ [28] producing a sharp minimum in reflection and a broadening and slight blue-shifting of the dominant dipole resonance. This resonance associated with interference between the bright dipole mode where inphase currents are excited on each antenna and a dark mode with the top and bottom wire supporting in-phase currents and the central wire carrying a current out-of-phase with the other two. Fig. 2(b) shows the amplitude and phase of the current excitations on each wire. Away from the Fano resonance (and in the absence of diffraction), the currents on all wires are in phase. Near the resonance, however, not only is there a distinct maximum seen in the current amplitudes where, due to the symmetry of the geometry and illumination, the top and bottom wire have identical amplitudes and phases, it is clear there is a $\pi$ phase difference between the currents in the outer two wires and that of the current in the central wire. This feature is an example of a nanophotonic analog of Electromagnetically Induced Transparency (EIT) [29].
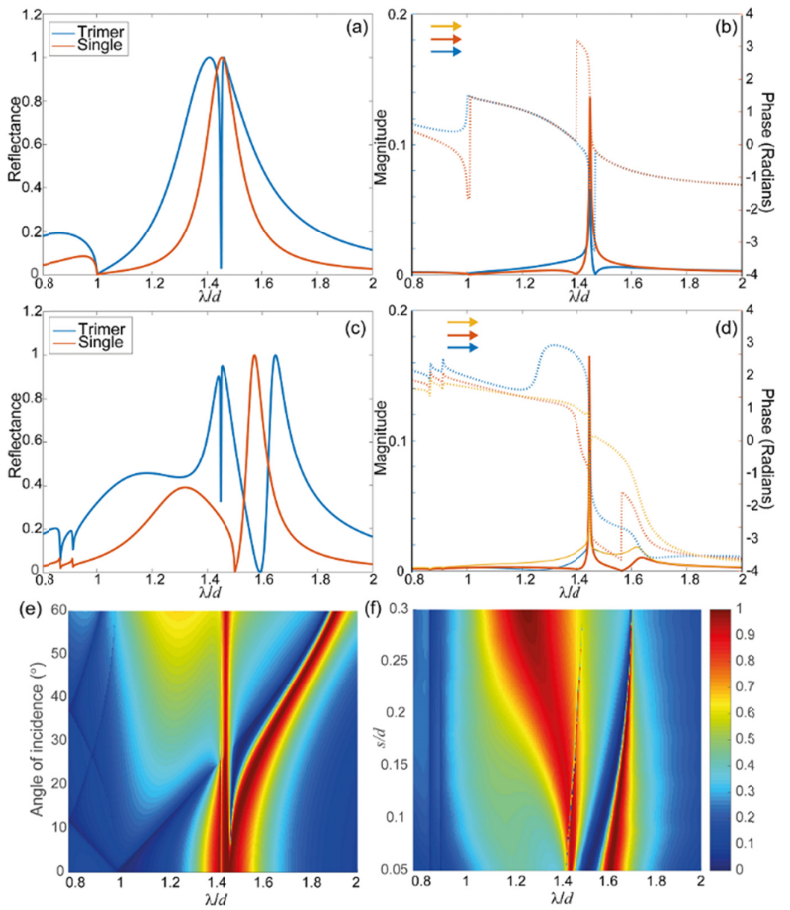

Fig. 2. Calculated reflection spectra from an array of parallel trimers of identical antennas of length $0.7 d$, width $0.02 d$ that are separated by a distance $s$ of $0.05 d$. (a) shows the reflectance at normal incidence and (b) the magnitude (solid lines) and phase (dotted lines) of the currents on each of the wires when the incident polarization is parallel to the wires. Figures (c) and (d) show the same results for $30^{\circ}$ incidence for TE-polarization. The results of the same calculations for a single dipole are also included in (a) and (c) for comparison and the insets in (b) and (d) show the direction of the zero-phase direction of the current. The reflectance as a function of wavelength and angle of incidence for TE-polarization is shown in (e) and (f) shows the reflectance as a function of wire separation for a fixed angle of incidence of $30^{\circ}$.

As the angle of incidence is increased to $30^{\circ}$, maintaining polarization parallel to the wires and TE-polarization (Fig. 2(c)), another mode appears in the reflectance spectrum with a maximum near $1.65 \mathrm{~d}$. Near this resonance, at a wavelength of $1.56 d$, the current in the central wire drops to zero and the phase difference between the currents in the top and bottom wires is close to $\pi$. This is consistent with the appearance of a magnetic mode accompanied by circulating, azimuthal electric fields. The presence of Wood anomalies associated with the transition of plane waves from propagating to evanescent is also apparent. For example, at normal incidence, (Fig. 2(a)), an 
anomaly appears at $\lambda / d=1$ where the $( \pm 1,0)$ and $(0, \pm 1)$ propagate parallel to the $x-y$ plane.

It is very apparent that by changing either the illumination or the geometry that a rich variety of resonance spectra can be generated. A plot showing the complex evolution of the reflectance spectrum with a change in the angle of incidence is shown in Fig. 2(e) and with a variation in the separation between the wires is shown in Fig. 2(f) for a fixed angle of incidence of $30^{\circ}$, maintaining TE-polarization parallel to the wires. In Fig. 2(e), the variation in the position of the Wood anomalies is immediately apparent as is the red-shifting of the magnetic mode with increasing angle of incidence, while the wavelength of the dipole resonance approaches that for the single wire. Changing the spacing between the wires also significantly modifies the reflectance spectrum. The spectral location of the Fano resonance depends only weakly on the separation between the wires, but slightly red-shifts. The dipole mode broadens and blue-shifts with changes in the difference in the phase of excitation of each wire.
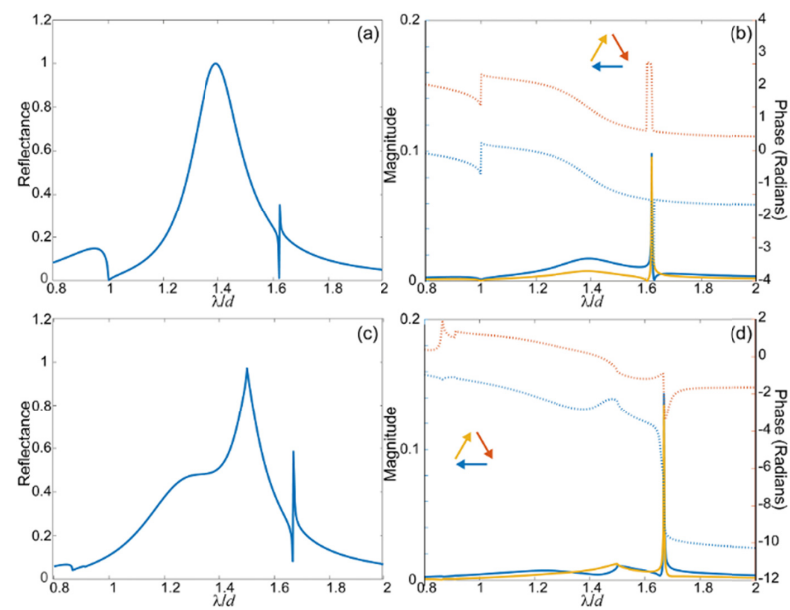

Fig. 3. Reflection from an array of identical wires of length, $a$, of $0.4 d$, in a triangular configuration with a distance from the center of each wire to the centroid of the arrangement, $s$, of $0.25 d$. (a) and (c) show the reflectance and (b) and (d) the amplitude (solid line) and phase (dashed line) of the current on each wire at (a) and (b) normal incidence and, (c) and (d) at an angle of incidence of $30^{\circ}$ assuming TE-polarization and parallel to one of the wires and the side of the unit cell.

As a further example, we look at reflection from arrays of three wires now arranged in a triangular configuration (Fig. 1(c)). Fig. 3 shows the normalized reflectance from such an array with $a=0.7 d$ and $b=0.02 d$ and with a fixed distance from the center of each wire to the center of the ensemble, $s$, of $0.25 d$. Results obtained at normal incidence (Fig. 3(a)) and for TEpolarization at $30^{\circ}$ incidence (Fig. 3(c)) are shown. In both cases the incident polarization is parallel to one of the wires and the axis of periodicity of the array. The magnitude and phase of the current on each of the wires for normal and off-normal incidence are shown in Figs. 3(b) and (d). Due to symmetry, the currents in the two upper wires are identical for both the illumination conditions considered. In both Fig. 3(a) and (c) Fano resonances at wavelengths of 1.62d (Fig. 3(a)) and 1.66d (Fig. 3(c)) respectively are apparent. From Fig. 3(b), it is clear that at normal incidence (according to the assumed current directions defined in the inset of Figs. 3(b) and (d)) the lower wire is out of phase with the upper wires away from the resonance. This is consistent with the excitation of a dipole mode of the ensemble. The Fano resonance is accompanied by maxima in the amplitudes of the currents on each of the wires and significant variations in the phase. At $30^{\circ}$ incidence (Fig. $3(d)$ ), a phase difference between the upper wires and the lower wire of approximately $\pi$ can be seen at shorter wavelengths consistent with the reflectance dominated by the ensemble dipole mode. At wavelengths longer than approximately $1.7 d$, however, the phase difference between the lower wire and the upper wires is approximately $2 \pi$ indicating the currents follow the sense defined in the inset suggesting a circulating magnetic mode. This mode is not excited at normal incidence.
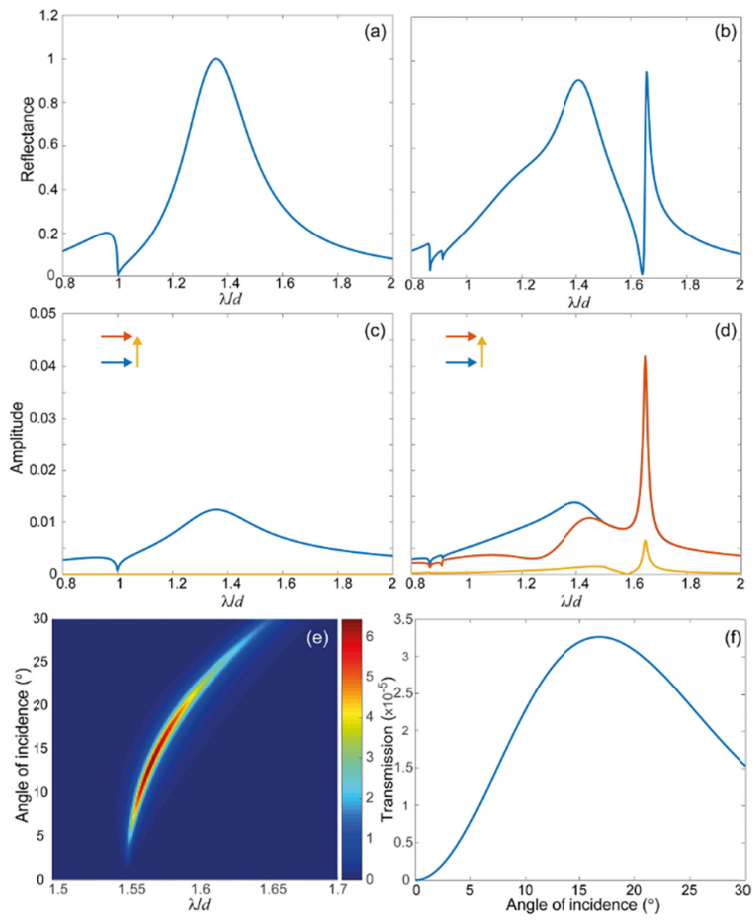

Fig. 4. Reflection from an array of trimers of three identical wires arranged as shown in Fig. 1(d) with length, $a, 0.7 d$, and a distance between the upper and lower wires of $0.68 d$. The gap, $\delta$, is fixed at $0.1 d$. (a) and (b) show the reflectance and (c) and (d) the amplitude and phase of the current on each wire at (a) and (c) normal incidence and, (b) and (d) at an angle of incidence of $30^{\circ}$ assuming $s$ polarization and incident polarization parallel to one of the wires and the side of the unit cell. The reflectance spectrum of the crosspolarized ( $\mathrm{y}$-) component of the transmitted power as a function of angle of incidence is shown in (e) and the average cross-polarized intensity transmitted between wavelengths of $1.5 d$ and $1.7 d$ is plotted in (f).

As a final example, we look at the dolmen [9] arrangement consisting of the three wires arranged in a U-shaped configuration as shown in Fig. 1(d) illuminated with a plane wave polarized parallel to the horizontal pair of wires. Here we again take $a=0.7 d$ and $b=0.02 d$, with a distance between the upper and lower wires, $s$, of $0.68 d$ and the gap defined in Fig. $1(d), \delta$, is fixed at $0.1 d$. It was shown recently [16] that if the arrangement is illuminated with an off-normal plane wave, TEpolarized with electric field parallel to the pair of wires then a cross-polarized component in the transmitted intensity if 
produced that is sensitive to angle of incidence through the phase gradient of the excitation field. The reflectance spectra for a plane wave at normal and at $30^{\circ}$ to normal incidence are shown in Fig. 4(a) and 4(c) respectively. In Fig. 4(a), a broad peak in reflectance centered on a wavelength of $1.36 d$ is apparent corresponding to excitation of the horizontal dipole mode of the ensemble. The amplitudes shown in Fig. 4(b) confirm that no current is excited on the vertical (as defined in the inset) wire. As the angle of incidence increases to $30^{\circ}$ (TEpolarization), a Fano resonance in the reflectance spectrum (Fig. 4(c)) appears at 1.65d. Looking at the amplitudes of the current on the wires as defined in the inset (Fig. 4(d)), it is apparent that the longer wavelength resonance is associated with excitation of current on the vertical wire. This excitation introduces a crosspolarized component into both the reflected and transmitted fields. To further highlight this, Fig. 4(e) shows the reflectance spectrum of the cross-polarized $(y-)$ component of the transmitted power as a function of angle of incidence. The feature in the spectrum near a wavelength of $1.55 \mathrm{~d}$ is associated with excitation of a current on the $y$-oriented dipole and increases with angle of incidence up to an angle of about $15^{\circ}$ beyond which the Wood anomaly red-shifts this resonance and reduces its strength. Fig. 4(f) shows the average cross-polarized intensity transmitted through a bandwidth delimited by wavelengths of $1.5 d$ and $1.7 d$ as a function of angle of incidence. Despite the small transmission, the dependence on spatial frequency is apparent. Tests indicate that the calculated spectral location of the peak transmission is (within the stated approximations) is accurate to better than $1 \%$.

In conclusion, dark modes of arrangements of dipoles forming the unit cell of a metasurface exhibit a wavelength-dependent sensitivity to angle of incidence. Three examples of trimeric metasurfaces were considered and their reflectance properties calculated. The sensitivity of these modes to angle of incidence and the associated phase gradient in the incident field raises the prospect of their use in various information processing applications [30]. The periodicity of the surface is integrated into the model so Wood anomalies associated with diffractive effects and their interference with localized resonances can also be seen. Furthermore, interference between radiant and subradiant modes producing Fano resonances lead to rich and complex spectra that can be tailored to create surfaces with specific properties for use as sensing elements, filters and other optical devices. Although quantitative predictions arising from this research are confined to the millimeter wave part of the spectrum, where assumptions about the high conductivity of metals is reasonable, the strategy presented here provides an intuitive approach to designing metasurfaces across the electromagnetic spectrum. Any results involving real materials and structures would need to be confirmed with a full field solver, but approximate, quasi-analytic approaches such as the electrostatic approximation and the methods proposed here, form the basis for rapid testing of concepts and structures.

Funding. Australian Research Council Discovery Projects (DP160100983) and Future Fellowship scheme (FT140100514)

\section{References}

1. S. A. Maier, Plasmonics: Fundamentals and Applications (Springer, New York, 2007).

2. A. V. Kildishev, A. Boltasseva, and V. M. Shalaev, "Planar photonics with metasurfaces," Science 339, 1232009 (2013).
3. N. Yu and F. Capasso, "Flat optics with designer metasurfaces," Nature Mater 13, 139-150 (2014)

4. R. Adato, A. A. Yanik, J. J. Amsden, D. L. Kaplan, F. G. Omenetto, M. K. Hong, S. Erramilli, and H. Altug, "Ultra-sensitive vibrational spectroscopy of protein monolayers with plasmonic nanoantenna arrays," Proc Natl Acad Sci U S A 106, 19227-19232 (2009).

5. M. W. Chu, V. Myroshnychenko, C. H. Chen, J. P. Deng, C. Y. Mou, and F. J. Garcia de Abajo, "Probing bright and dark surface-plasmon modes in individual and coupled noble metal nanoparticles using an electron beam," Nano Lett 9, 399-404 (2009).

6. B. K. Canfield, H. Husu, J. Laukkanen, B. Bai, M. Kuittinen, J. Turunen, and M. Kauranen, "Local Field Asymmetry Drives Second-Harmonic Generation in Noncentrosymmetric Nanodimers," Nano Lett 7, 12511255 (2007)

7. D. E. Gomez, A. Roberts, T. J. Davis, and K. C. Vernon, "Surface plasmon hybridization and exciton coupling," Phys Rev B 86(2012).

8. T. J. Davis, D. Gomez, and K. C. Vernon, "Simple Model for the Hybridization of Surface Plasmon Resonances in Metallic Nanoparticles," Nano Lett 10, 2618-2625 (2010).

9. T. J. Davis, K. C. Vernon, and D. E. Gomez, "A plasmonic "ac Wheatstone bridge" circuit for high-sensitivity phase measurement and singlemolecule detection," J Appl Phys 106, 043502 (2009).

10. T. J. Davis, K. C. Vernon, and D. E. Gomez, "Designing plasmonic systems using optical coupling between nanoparticles," Phys Rev B 79, 155423-155421-155410 (2009).

11. T. J. Davis, M. Hentschel, N. Liu, and H. Giessen, "Analytical Model of the Three-Dimensional Plasmonic Ruler," ACS Nano 6, 1291-1298 (2012).

12. N. Liu, M. Hentschel, T. Weiss, A. P. Alivisatos, and H. Giessen, "ThreeDimensional Plasmon Rulers," Science 332, 1407 (2011).

13. B. Munk, Frequency Selective Surfaces: Theory and Design (John Wiley and Sons, New York, 200).

14. R. Mittra, C. H. Chan, and T. Cwik, "Techniques for analyzing frequency selective surfaces-a review," Proc IEEE 76, 1593-1615 (1988).

15. T.-K. Wu, "Frequency Selective Surfaces," in Encyclopedia of RF and Microwave Engineering (John Wiley and Sons, New York, 2005).

16. E. Eftekhari, D. Gómez, and T. Davis, "Measuring subwavelength phase differences with a plasmonic circuit--an example of nanoscale optical signal processing," Opt Lett 39, 2994-2997 (2014).

17. Y. Hwang and T. J. Davis, "Optical metasurfaces for subwavelength difference operations," Appl Phys Lett 109, 181101 (2016).

18. V. A. Fedotov, J. Wallauer, M. Walther, M. Perino, N. Papasimakis, and N. I. Zheludev, "Wavevector Selective Metasurfaces and Tunnel Vision Filters," Light: Science \& Applications 4, e306 (2015).

19. T. Wei and S. Zhongxiang, "Scattering by a two-dimensional periodic array of multiple plates," in 2007 IEEE Antennas and Propagation Society International Symposium, (IEEE, 2007), 4533-4536.

20. S. H. Mousavi, A. B. Khanikaev, and G. Shvets, "Optical properties of Fano-resonant metallic metasurfaces on a substrate," Phys Rev B 85, 155429 (2012).

21. C. Chao-Chun, "Scattering by a two-dimensional periodic array of conducting plates," IEEE Transactions on Antennas and Propagation 18, 660-665 (1970).

22. C.-C. Chen, "Transmission of Microwaves Through Perforated Flat Plates of Finite Thickness," IEEE Trans Microw Theory Tech 21, 1-6 (1973).

23. C.-C. Chen, "Diffraction of Electromagnetic Waves by a Conducting Screen Perforated Periodically with Circular Holes," IEEE Trans Microw Theory Tech 19, 475-481 (1971).

24. D. H. Dawes, R. C. McPhedran, and L. B. Whitbourn, "Thin capacitive meshes on a dielectric boundary: theory and experiment," Appl Opt 28 3498-3510 (1989).

25. J. Montgomery, "Scattering by an infinite periodic array of thin conductors on a dielectric sheet," IEEE Transactions on Antennas and Propagation 23, 70-75 (1975).

26. L. B. Whitbourn and R. C. Compton, "Equivalent-circuit forumulas for metal grid reflectors at a dielectric boundary," Appl Opt 24, 217-220 (1985). 
27. R. C. Compton, "Approximation Techniques for Planar Periodic Structures," IEEE Trans Microw Theory Tech 33, 1083-1088 (1985).

28. Z.-L. Deng, T. Fu, Z. Ouyang, and G. Wang, "Trimeric metasurfaces for independent control of bright and dark modes of Fano resonances," Appl Phys Lett 108, 81109 (2016).

29. S. Zhang, D. A. Genov, Y. Wang, M. Liu, and X. Zhang, "PlasmonInduced Transparency in Metamaterials," Phys Rev Lett 101, 047401 (2008).

30. A. Silva, F. Monticone, G. Castaldi, V. Galdi, A. Alu, and N. Engheta, "Performing Mathematical Operations with Metamaterials," Science 343, 160-163 (2014) 